\title{
Interleukin-2-Inducible T-Cell Kinase Deficiency-New Patients, New Insight?
}

\author{
Sujal Ghosh ${ }^{1 *}$, Ingo Drexler², Sanil Bhatia', Heiko Adler ${ }^{3,4,5}$, Andrew R. Gennery ${ }^{6}$ and Arndt \\ Borkhardt ${ }^{1}$
}

\begin{abstract}
1 Department of Pediatric Oncology, Hematology and Clinical Immunology, Medical Faculty, Center of Child and Adolescent Health, Heinrich-Heine-University, Düsseldorf, Germany, ${ }^{2}$ Institute for Virology, Medical Faculty, Heinrich-Heine-Universität Düsseldorf, Düsseldorf, Germany, ${ }^{3}$ Research Unit Lung Repair and Regeneration, Comprehensive Pneumology Center, Helmholtz Zentrum München-Deutsches Forschungszentrum für Gesundheit und Umwelt (GmbH), Munich, Germany, ${ }^{4}$ University Hospital Grosshadern, Ludwig-Maximilians-Universität München, Munich, Germany, ${ }^{5}$ German Center for Lung Research (DZL), Giessen, Germany, ${ }^{6}$ Paediatric Immunology and HSCT, Newcastle University and Great North Children's Hospital, Newcastle upon Tyne, United Kingdom
\end{abstract}

\section{OPEN ACCESS}

Edited by:

Isabelle Meyts,

KU Leuven, Belgium

Reviewed by:

Stuart G. Tangye,

Garvan Institute of Medical

Research, Australia

Shigeaki Nonoyama,

National Defense Medical

College, Japan

Pamela Lee Schwartzberg,

National Institutes of Health

$(\mathrm{NIH})$, United States

*Correspondence:

Sujal Ghosh

sujal.ghosh@med.

uni-duesseldorf.de

Specialty section: This article was submitted to Primary Immunodeficiencies,

a section of the journal

Frontiers in Immunology

Received: 23 October 2017 Accepted: 20 April 2018

Published: 08 May 2018

Citation:

Ghosh S, Drexler I, Bhatia S, Adler H, Gennery AR and Borkhardt $A$ (2018) Interleukin-2-Inducible

T-Cell Kinase Deficiency-

New Patients, New Insight?

Front. Immunol. 9:979.

doi: 10.3389/fimmu.2018.00979
Patients with primary immunodeficiency can be prone to severe Epstein-Barr virus (EBV) associated immune dysregulation. Individuals with mutations in the interleukin-2inducible T-cell kinase (ITK) gene experience Hodgkin and non-Hodgkin lymphoma, EBV lymphoproliferative disease, hemophagocytic lymphohistiocytosis, and dysgammaglobulinemia. In this review, we give an update on further reported patients. We believe that current clinical data advocate early definitive treatment by hematopoietic stem cell transplantation, as transplant outcome in primary immunodeficiency disorders in general has gradually improved in recent years. Furthermore, we summarize experimental data in the murine model to provide further insight of pathophysiology in ITK deficiency.

Keywords: primary immunodeficiency, combined immunodeficiency, interleukin-2-inducible T-cell kinase, Epstein-Barr virus-related malignancies, lymphoproliferative disorders

\section{INTRODUCTION}

Epstein-Barr virus (EBV) is recognized to cause infectious mononucleosis. More than $90 \%$ of the global population carries the latent virus life-long and most individuals acquire the gammaherpesvirus by silent infection at young age. Several malignancies are associated with EBV and in the last decades patients with genetic defects of $\mathrm{T}$ cell signaling or cytotoxic pathway have demonstrated susceptibility to severe immune dysregulation upon EBV infection or reactivation. They usually present with fatal infectious mononucleosis, lymphoma and lymphoproliferative disease (LPD), hemophagocytic lymphohistiocytosis (HLH), and dysgammaglobulinemia $(1,2)$.

While many combined immunodeficiencies (e.g., defects of antigen receptor recombination $R A G 1 / 2)$ can lead to EBV immune dysregulation beside other infectious complications, there are diseases, which confer a higher propensity only of EBV associated disease. Several genes have been linked to EBV lymphoproliferation (SH2D1A, STK4, CD27, CD70, LAT, RASGRP1, MAGT1, Coronin-1A, and CTPS1) in recent years (2). Our group and others reported alterations in the interleukin-2-inducible T-cell kinase (ITK) gene in patients presenting with severe EBV associated dysregulation $(3,4)$. At least one decade earlier, murine studies had already shown that ITK is essential for various $\mathrm{T}$ cell functions, especially during a Th2 response. In this mini review, we update on clinical and immunological aspects in reported individuals and highlight the extensively investigated murine itk-/- model. 


\section{ITK DEFICIENCY - CLINICAL PRESENTATION AND DIAGNOSIS}

The first patients were reported in 2009 by our group. Two sisters from consanguineous Turkish parents presented with EBVdriven lymphoproliferative disease (3). At age of 6 years, one developed pneumocystis pneumonitis, severe candida stomatitis, cytopenia, progressive hypogammaglobulinemia, and oligoclonal polymorphic B cell lymphoproliferation.

Eighteen months later, she presented with Hodgkin lymphoma (HL), which was successfully treated with chemotherapy. However, $\mathrm{T}$ lymphocytes were further declining and at age of 10 years the girl succumbed to pneumocystis pneumonia. The younger sister presented with pancytopenia and severely impaired hepatic function due to EBV-associated HL. Due to rapid clinical deterioration haploidentical peripheral blood stem cell transplantation (SCT) was performed as a salvage therapy, but unfortunately the patient died due to airway obstruction during aplasia. Genome-wide linkage analysis identified ITK, in which the causative homozygous R335W mutation was revealed. To date, we are aware of ITK mutations in 17 patients originating from Greece, India, Italy, Iran, Morocco, Pakistan, Palestine, and Turkey (16 patients described in Table 1) (5-12). These patients manifested between 2.5 months and 13 years of age and presented with fever, hepatosplenomegaly, lymphadenopathy, and EBV viremia. One patient was diagnosed at birth due to family history of disease in the older brother. Thirteen patients presented either with HL or with EBV-driven B cell lymphoproliferative disease (in some cases developing to Hodgkin or large B cell lymphoma), only two showed a classical non-HL histology. In a few patients, other viral infections including CMV and VZV were seen. Given the severe immune dysregulation, at least three patients developed autoimmune phenomena and two patients developed HLH.

The number of ITK patients is too few to deduce valid statistics. However, it appears that HLH occurs less frequent in ITK deficiency than, e.g., in SLAM-associated protein (SAP) deficiency (30\%) (13).

As known from other disorders with EBV predisposition, pulmonary interstitial nodules were seen in most patients. Furthermore, progressive hypogammaglobulinemia and loss of CD4+ T cells was detected, in particular naive CD45RA+ CD4+ $T$ cells were decreased. In parallel with other EBV prone disorders (e.g., SAP deficiency), peripheral NKT cells [determined as CD3+, T cell receptor (TCR) Vbeta11+, TCR Valpha24+] were decreased in ITK-deficient patients supporting observations in transformed cell lines that NKT cells might be essential for anti EBV immunity (14). However, there is some evidence that EBV infection itself might decrease the number of NKT cells in these patients, as normal numbers of NKT cells are demonstrated in EBV-naive patients, e.g. in patients with XIAP deficiency (15). Furthermore, there are disorders with a global lack of NKT cells, in which individuals are rather susceptible to Mycobacteria, but not to EBV infection (16).

Peak EBV viremia in ITK-deficient patients was quite heterogeneous in reported patients $\left(10^{4}-10^{8}\right.$ copies/ $\mu$ g DNA). Unfortunately, we obtained incomplete information on serological phenotype at time of manifestation to predict the time between infection and clinical exacerbation; EBV-VCA-IgM was detected in one patient only. In contrast to one of the most similar immunological disorders-SAP deficiency - there is not a single reported EBV-VCA-IgG seronegative symptomatic EBV-LPD patient highlighting the paramount importance of EBV infection and maybe specificity in the disease setting. Interestingly the spectrum of histopathological diagnosis is quite variable in reported patients. Bienemann et al analyzed seven of the 16 patients presented here. In six events, a classic mixed-cellular HL histology was shown, while the other lymphoproliferative events were rather heterogeneous (polymorphic: three events, borderline polymorphic to monomorphic blast-rich B-cell LPD: two events, HL-like B-cell proliferation: two events and large B-cell lymphoma like LPD: two events). In contrast to many immunocompromised patients (who rather demonstrate latency type III), ITK-deficient patients had predominantly EBV latency type II and presented often with nodal and extranodal manifestations simultaneously (6). One patient with ITK deficiency differs from the other patients in several points. An 18-year-old male Turkish patient suffered from recurrent progressive pulmonary infections and bronchiectasis, but no lymphoproliferative disease. He remained EBV seronegative although PCR could detect a low EBV viral load of 1,000-2,000 copies/ $\mu$ l (11).

\section{MANAGEMENT AND OUTCOME}

As previously demonstrated in other EBV-LPD cases, a few patients with ITK deficiency were treated with Rituximab with some improvement. IgG substitution has conferred only temporary benefit, especially to partially ameliorate immune dysregulation manifesting as lymphoproliferation and autoimmunity; corticosteroids were not helpful in the reported cases. Eight patients died between 1 and 15 years after diagnosis (mostly due to malignancies), seven within 2 years from diagnosis. Nine patients did not receive definitive treatment. Most had a fatal outcome. Six patients died due to lymphoproliferation, while only two patients remained in remission after chemotherapy for HL. However, eight patients underwent hematopoietic SCT. Two patients died after HSCT. While one of the initial patients died during aplasia with hemorrhagic acute airway obstruction after receiving haploidentical PBSCT, another patient succumbed to severe graft-versus-host disease. Recently, three more patients have been reported at two different centers (Newcastle, UK and Paris, France), which have been presented orally at the Annual Meeting of the European Society for Immunodeficiencies in Edinburgh, September 2017. All three patients were diagnosed with Hodgkin-like lymphoma or diffuse B cell lymphoma like lymphoproliferation and were subject to HSCT. Remarkably, the Paris patient was treated with five courses of Rituximab and two injections of Brentuximab to achieve clinical remission before haploidentical T replete HSCT. We can learn from those cases that immunotherapy with Rituximab or Brentuximab can lead to partial or even complete remission and at least bridge to definitive cure. We strongly suggest that each patient should be carefully considered for early HSCT, once the diagnosis of ITK deficiency has been established. 


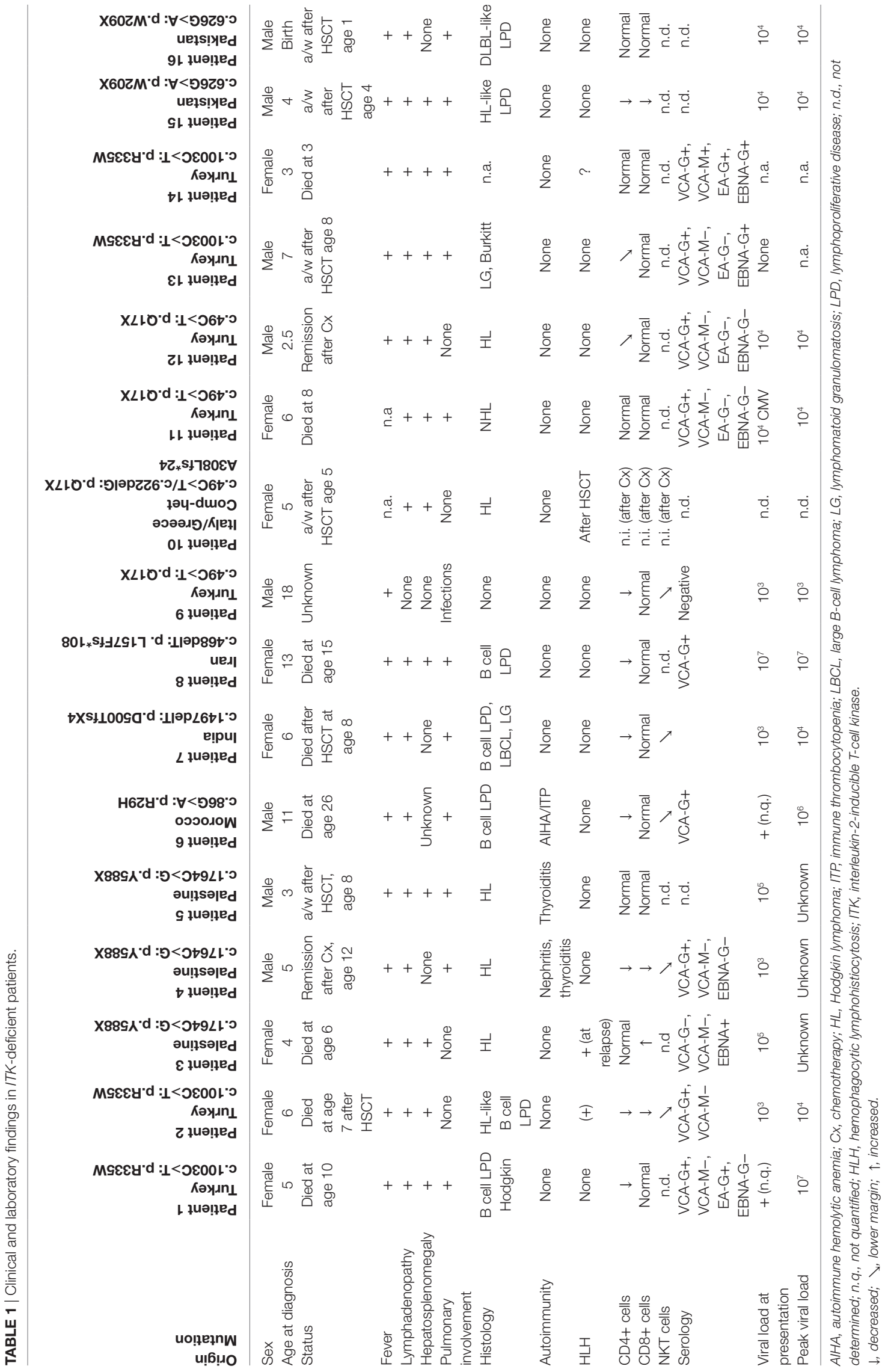




\section{INTERLEUKIN-2-INDUCIBLE T-CELL KINASE}

Interleukin-2-inducible T-cell kinase is one of five mammal TEC family kinases. All five proteins are involved in lymphocyte signaling and development (17). Years before the first patient with ITK deficiency was diagnosed, ITK-SYK translocations were found in individuals with T cell lymphoma (18). The ITK gene on chromosome $5 \mathrm{q}$ consists of 17 exons and $112 \mathrm{kbp}$, the protein $(71 \mathrm{kDa})$ is formed by 620 amino acids. ITK is composed of an N-terminal pleckstrin homology (PH), a Tec homology
(TH), an Src homology 3 (SH3), an Src homology 2 (SH2), and a C-terminal catalytic kinase domain (Figure 1A) (19). Upon activation of the TCR several phosphorylation events recruit ITK to the cell membrane (for details, see Figure 1B). ITK activates PLC $\gamma 1$, generating inositol 1,4,5-trisphosphate (IP3), which leads to intracellular calcium release and diacylglycerol, which, via RASGRP and PKC $\delta$, ultimately results in activation/induction of the NFKB, mTOR, and MAPK/ERK pathways.

Mutations were found in the kinase, $\mathrm{SH} 2$ and $\mathrm{PH}$ domain. Most patients demonstrated an autosomal-recessive trait, while in one individual a compound-heterozygous inheritance from

\section{A}

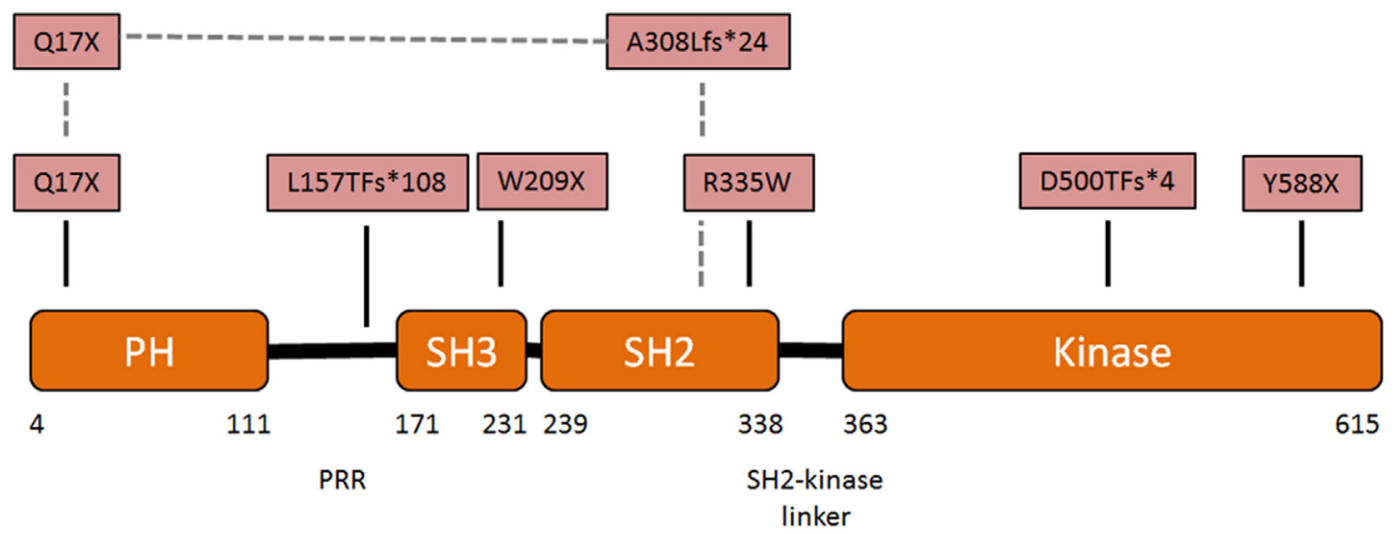

B

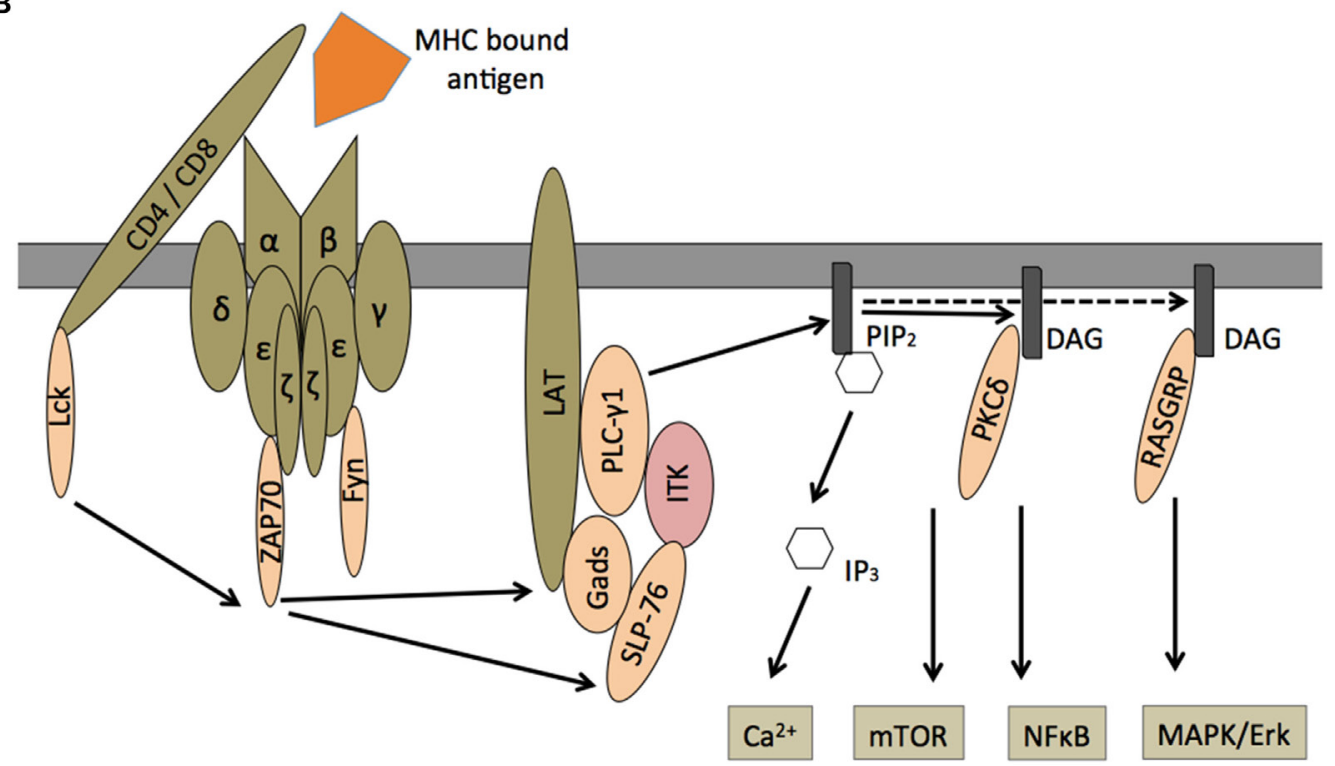

FIGURE 1 | Interleukin-2-inducible T-cell kinase (ITK) -structure and signaling - (A) domain organization of ITK and corresponding protein mutants in patients with ITK deficiency. N-terminal pleckstrin homology (PH), Tec homology, Src homology 3 (SH3), Src homology 2 (SH2), and C-terminal catalytic kinase domain. Pattern recognition receptors. In one patient, a compound heterozygous mutation is predicted to encode Q17X and A308LfS*24 mutant. (B) Following engagement of the T cell receptor (TCR) with an MHC bound foreign antigen, several intracellular signals are activated. Lck is recruited and phosphorylates immunoreceptor tyrosinebased activation motifs (ITAM) at the zeta chain of the TCR. ZAP70 binds double-phosphorylated ITAM residues and phosphorylates LAT, which is recruited to the TCR complex. Phosphorylated LAT recruits SLP76, which together with Itk, activates PLC $\gamma 1$. Subsequently phosphatidylinositol 4,5-bisphosphate (PIP2) is catalyzed into inositol 1,4,5-trisphosphate (IP3), which leads to intracellular calcium release and diacylglycerol (DAG). DAG itself can recruit PKC $\delta$ and RASGRP, which induce the NFKB and MAPK/ERK pathways. 
two different ethnicities (Greek-Italian) was revealed (5). Interestingly there are corresponding mutations in residues of the "sister" Tec kinase BTK (known to cause X-linked agammaglobulinemia), which are homologous to the mutations observed in our patients (9). Our group transformed Herpesvirus saimiri cell lines to reveal functional impairment in corresponding ITK mutations.

The alterations did not greatly change the amount of ITK mRNA expression, nevertheless immunoblot investigations showed several variants of endogenous ITK. Most importantly, we analyzed calcium response with flow cytometric flux studies and revealed a highly decreased release of calcium ions into the cytosol in most patients. With regards to functional complementation our group restored TCR-mediated calcium flux in murine $i t k-/-$ thymocytes by means of wild type ITK transduction.

Interestingly since the publication of our last review two new EBV prone diseases have been discovered in the proximity of ITK (with respect to location in the pathway). Linker for activation of T cells (LAT) is a transmembrane adapter molecule, which is phosphorylated after TCR triggering. It contains no intrinsic enzymatic activity and couples the TCR to downstream pathways as a scaffolding protein. PLCy1 phosphorylation is highly dependent on the assembly of the LAT-SLP76 signalosome. However, the two initial reports on two kindreds with LAT deficiency show different phenotypes and ambiguous results $(20,21)$. One kindred presented with a typical (severe) immunodeficiency phenotype with failure to thrive and recurrent infections. The other report describes a family with infants with increased autoimmunity due to combined immunodeficiency with a higher number of residual T lymphocytes. All three siblings suffered from CMV and EBV infection before autoimmunity developed. Similar to our investigations in ITK-deficient cell lines, both of these new reports demonstrate decreased $\mathrm{Ca} 2+$ mobilization and other downstream signaling in LAT-deficient Jurkat cell lines (J.CaM2.5, ANJ3), and although, ITK phosphorylation of ITK, is reported to be dependent on LAT, it was not affected in J.CaM2.5. However, the same group (with the higher number of residual $\mathrm{T}$ lymphocytes and autoimmunity) had the opportunity to examine calcium flux in CD45RO patient lymphocytes, which was surprisingly within the range of healthy controls 21 . Interestingly, all these patients had infectious (often CMV-relate) and autoimmune problems, rather than emerging lymphoproliferation. RASGRP1 is a guanine nucleotide exchange factor, which is downstream of the PLC $\gamma 1$ mediated cleavage of phosphatidylinositol 4,5-bisphosphate. Mutations in RASGRP1 have also been associated with CD4 T lymphopenia, EBV-driven B cell lymphoma, and lymphoproliferative disease $(22,23)$.

\section{Itk-/- MURINE PHENOTYPE}

The murine itk-/- phenotype has been investigated for more than 25 years now, 15 years before the first patients were reported. Most studies had focused on Th1 skewing especially in infectious models; recent data further suggests that itk-/- CTLs harbor defects in expansion, degranulation and thus cytotoxicity. In the next chapter we will briefly summarize the itk-/- murine phenotype.
Itk-/- mice show an altered development of thymocytes with elevated numbers of innate single positive CD8+ (CD8SP) cells. These thymocytes parallel antigen-experienced $\mathrm{T}$ cells with a CD122+ CD44hiCXCR3+ phenotype and increased production of Eomesodermin and IFN $\gamma$, if stimulated. Similarly splenocytes (having decreased CD4 and CD8 expression in total) resemble a more differentiated phenotype (CD44+) mirroring peripheral CD8 cells of ITK-deficient patients (24-29). NKT cells have an impaired development, are dysfunctional and have a decreased survival in the periphery (30). Most studies, addressing the Th1 and Th2 paradigm suggest that ITK plays a role in a correct Th2 response (19). Upon TCR stimulation, itk-/- T cells have an impaired proliferation, less intracellular calcium release and a reduced production of effector cytokines (31).

Few epidemiological studies have observed asthma predisposition and variants in the ITK gene $(32,33)$. Several papers investigated the $\mathrm{T}$ lymphocytes dependent airway hyporesponsiveness in itk-/- mice. Pathophysiology of asthma usually involves pulmonary infiltration of Th2 cells. Due to an impaired Th2 response itk-/- mice show a reduced airway inflammation upon challenge with allergens $(32,34,35)$. One group tried to administer an ITK inhibitor as a pharmacologic agent to suppress inflammation in already ovalbumin-induced hyperresponsive airways. Paradoxically, inhibition of ITK induced lymphoid hyperplasia, an observation they attributed to impaired cell death in the absence of cell death (32). Two studies have further focused on the impaired cell death in itk-/- mice, which might be at least one explanation for the lymphoproliferation seen in the patients. One study found reduced activation-induced cell death, evidenced by defective FasL upregulation upon activation and elevated T cell proliferation (36).

In recent years, Th17, Treg, and Th9 differentiation have been extensively addressed as well (37-39).

Infections show the impact of ITK on T cell differentiation and $T$ cell effector function. In one of the first studies itk-/- mice on a BALB/c background failed to generate the usual Th2 response upon infection with Leishmania major, but rather mounted a Th1 dependent IFN $\gamma$ response, which cleared the infection (31). In further studies itk-/- mice showed decreased granuloma formation after challenge with Schistosoma mansoni eggs or the nematode Nippostrongylus brasiliensis. Both helminths usually induce a Th2 response $(31,40)$. Upon S. mansoni infection compared to WT the size of granuloma and draining lymph nodes was significantly decreased and production of the Th2 cytokines IL-4, IL-5 and IL-10 was markedly reduced in itk-/- mice. Again, IFN $\gamma$ levels were significantly higher suggesting Th1 skewing. If infected with $N$. brasiliensis, wild type BALB/c mice were able to fight the intestinal infection, while itk-/- mice showed a decrease in IL-4 and were incapable to clear the worm.

Toxoplasma gondii, on the other hand, promotes Th1 mediatedimmunity. Although itk-/- mice do succumb to this infection, they are only slightly more susceptible to $T$. gondii than wild type mice (41). Serum IFN-y levels 5 days after infection and splenic IFN-y production upon stimulation after 30 days show similar values as wild type mice. Only few studies have addressed the CD8 T cell response in itk-/- mice. It was reported that, although itk-/- mice do mount protective responses to lymphocytic 
choriomeningitis virus Armstrong, vaccinia virus, and vesicular stomatitis virus, viral clearance is delayed, most likely due to poor activation of CD8 T lymphocytes $(42,43)$. Given the clinical phenotype of the reported patients, a potential role for ITK in CTL function seems highly likely. Recently, the effect of ITK on cytotoxicity and degranulation of CTLs was demonstrated. ITKdeficient CTLs showed decreased expansion and a more naïve phenotype after activation. The authors revealed that in murine itk-/- deficient lymphocytes, early stages of cytotoxicity were intact, while defects in degranulation were the bigger concern (44).

As far as we know there has not been any study in which an infection model of the murine gammaherpesvirus 68 (MHV-68) has been investigated in itk-/- mice, although murine MHV-68 infection resembles human EBV infection quite a bit. MHV-68 spreads naturally by the respiratory route and is genetically related to EBV (45). Both EBV and MHV-68 have the ability to cause infectious mononucleosis. Following intranasal inoculation the virus causes an acute infection in the lungs and remains in a latent form within B cells. Depending on CD8 T cell function, MHV-68 can further infect other splenic B cells and circulate in other organs. MHV-68 infection has already been investigated in SAP deficient mice (Sh2d1a-/-) leading to hypogammaglobulinemia and organ damage $(46,47)$. Clinically, patients with SAP deficiency have shared features with patients with ITK deficiency, hence we decided to explore the natural course of MHV-68 infection in $i t k-/-$ mice in some preliminary experiments. B6 and itk-/- mice were intranasally infected with MHV-68. There was no difference in the lytic viral tire in lungs between B6 and itk-/- infected mice; furthermore, there was no difference in the splenic genomic viral load between $\mathrm{B} 6$ and $i t k-/-$ mice at day 17 . Clinically the mice did not behave differently. Similarly to Sh2d1a-/- mice after 3 months in total itk-/- mice spleens were enlarged, and we could

\section{REFERENCES}

1. Parvaneh N, Filipovich AH, Borkhardt A. Primary immunodeficiencies predisposed to Epstein-Barr virus-driven haematological diseases. Br J Haematol (2013) 162:573-86. doi:10.1111/bjh.12422

2. Tangye SG, Palendira U, Edwards ES. Human immunity against EBV-lessons from the clinic. J Exp Med (2017) 214:269-83. doi:10.1084/jem.20161846

3. Huck K, Feyen O, Niehues T, Ruschendorf F, Hubner N, Laws HJ, et al. Girls homozygous for an IL-2-inducible T cell kinase mutation that leads to protein deficiency develop fatal EBV-associated lymphoproliferation. J Clin Invest (2009) 119:1350-8. doi:10.1172/JCI37901

4. Ghosh S, Bienemann K, Boztug K, Borkhardt A. Interleukin-2-inducible T-cell kinase (ITK) deficiency - clinical and molecular aspects. J Clin Immunol (2014) 34:892-9. doi:10.1007/s10875-014-0110-8

5. Alme C, Satwani P, Alobeid B, Bhagat G, Kelly KM. Atypical clinical course in pediatric Hodgkin lymphoma: association with germline mutations in interleukin-2-inducible T-cell kinase. J Pediatr Hematol Oncol (2015) 37: 507-8. doi:10.1097/MPH.0000000000000366

6. Bienemann K, Borkhardt A, Klapper W, Oschlies I. High incidence of EpsteinBarr virus (EBV)-positive Hodgkin lymphoma and Hodgkin lymphoma-like B-cell lymphoproliferations with EBV latency profile 2 in children with interleukin-2-inducible T-cell kinase deficiency. Histopathology (2015) 67:607-16. doi:10.1111/his.12677

7. Cagdas D, Erman B, Hanoglu D, Tavil B, Kuskonmaz B, Aydin B, et al. Course of IL-2-inducible T-cell kinase deficiency in a family: lymphomatoid granulomatosis, lymphoma and allogeneic bone marrow transplantation in verify a Vbeta4 expansion in all infected mice, similar to other mouse models after MHV-68 infection. Interestingly, we saw a relative decrease in CD4 cells in itk-/- mice; on the other hand, CD8 numbers were similar in both groups. Most importantly we saw a bigger expansion of Vbeta 4 cells within in the itk-/- group (own preliminary results). The expansion of this clone is line with reports in Sh2d1a-/- deficient mice, and further experiments are ongoing to evaluate a potential use of this model to investigate ITK deficiency.

\section{SUMMARY}

Since our last review the reported patient number with ITK deficiency has nearly doubled. All patients with previous EBV infection, developed EBV-associated malignancies, like Hodgkin and non-HL and lymphoproliferative diseases, while pulmonary involvement is one of the extranodal key features. Although the number of patients is limited, a curative treatment should be considered. In settings in which an HLA-matched donor is lacking, a haploidentical donor in conjunction with advanced T-depleting and adoptive T cell transfer strategies have improved the outcome. Immunotherapy with anti-CD20 or anti-CD30 can bridge to definitive cure. EBV-negative patients (without any viremia) have not been reported yet, so we are unaware of any problems in these individuals. However, an early transplant might improve outcome. Prospective data collection on HSCT in ITK deficiency and other EBV prone primary immunodeficiencies, as CD27 or CD70 deficiency is highly warranted.

\section{AUTHOR CONTRIBUTIONS}

All the authors wrote the manuscript and gathered data.

one sibling; and death in the other. Bone Marrow Transplant (2017) 52:126-9. doi:10.1038/bmt.2016.185

8. Cipe FE, Aydogmus C, Serwas NK, Tugcu D, Demirkaya M, Bicici FA, et al. ITK deficiency: how can EBV be treated before lymphoma? Pediatr Blood Cancer (2015) 62:2247-8. doi:10.1002/pbc.25648

9. Linka RM, Risse SL, Bienemann K, Werner M, Linka Y, Krux F, et al. Lossof-function mutations within the IL-2 inducible kinase ITK in patients with EBV-associated lymphoproliferative diseases. Leukemia (2012) 26:963-71. doi:10.1038/leu.2011.371

10. Mansouri D, Mahdaviani SA, Khalilzadeh S, Mohajerani SA, Hasanzad M, Sadr S, et al. IL-2-inducible T-cell kinase deficiency with pulmonary manifestations due to disseminated Epstein-Barr virus infection. Int Arch Allergy Immunol (2012) 158:418-22. doi:10.1159/000333472

11. Serwas NK, Cagdas D, Ban SA, Bienemann K, Salzer E, Tezcan I, et al. Identification of ITK deficiency as a novel genetic cause of idiopathic CD4+ T-cell lymphopenia. Blood (2014) 124:655-7. doi:10.1182/blood-2014-03-564930

12. Stepensky P, Weintraub M, Yanir A, Revel-Vilk S, Krux F, Huck K, et al. IL-2inducible T-cell kinase deficiency: clinical presentation and therapeutic approach.Haematologica(2011)96:472-6.doi:10.3324/haematol.2010.033910

13. Booth C, Gilmour KC, Veys P, Gennery AR, Slatter MA, Chapel H, et al. $\mathrm{X}$-linked lymphoproliferative disease due to SAP/SH2D1A deficiency: a multicenter study on the manifestations, management and outcome of the disease. Blood (2011) 117:53-62. doi:10.1182/blood-2010-06-284935

14. Chung BK, Tsai K, Allan LL, Zheng DJ, Nie JC, Biggs CM, et al. Innate immune control of EBV-infected B cells by invariant natural killer T cells. Blood (2013) 122:2600-8. doi:10.1182/blood-2013-01-480665 
15. Marsh RA, Madden L, Kitchen BJ, Mody R, McClimon B, Jordan MB, et al. XIAP deficiency: a unique primary immunodeficiency best classified as $\mathrm{X}$-linked familial hemophagocytic lymphohistiocytosis and not as X-linked lymphoproliferative disease. Blood (2010) 116:1079-82. doi:10.1182/blood2010-01-256099

16. Okada S, Markle JG, Deenick EK, Mele F, Averbuch D, Lagos M, et al. IMMUNODEFICIENCIES. Impairment of immunity to Candida and Mycobacterium in humans with bi-allelic RORC mutations. Science (2015) 349: 606-13. doi:10.1126/science.aaa4282

17. Horwood NJ, Urbaniak AM, Danks L. Tec family kinases in inflammation and disease. Int Rev Immunol (2012) 31:87-103. doi:10.3109/08830185. 2012.670334

18. Hussain A, Yu L, Faryal R, Mohammad DK, Mohamed AJ, Smith CI. TEC family kinases in health and disease - loss-of-function of BTK and ITK and the gain-of-function fusions ITK-SYK and BTK-SYK. FEBS J (2011) 278:2001-10. doi:10.1111/j.1742-4658.2011.08134.x

19. Kosaka Y, Felices M, Berg LJ. Itk and Th2 responses: action but no reaction. Trends Immunol (2006) 27:453-60. doi:10.1016/j.it.2006.08.006

20. Bacchelli C, Moretti FA, Carmo M, Adams S, Stanescu HC, Pearce K, et al. Mutations in linker for activation of T cells (LAT) lead to a novel form of severe combined immunodeficiency. J Allergy Clin Immunol (2017) 139(634-42): e5. doi:10.1016/j.jaci.2016.05.036

21. Keller B, Zaidman I, Yousefi OS, Hershkovitz D, Stein J, Unger S, et al. Early onset combined immunodeficiency and autoimmunity in patients with lossof-function mutation in LAT. J Exp Med (2016) 213:1185-99. doi:10.1084/ jem.20151110

22. Platt CD, Fried AJ, Hoyos-Bachiloglu R, Usmani GN, Schmidt B, Whangbo J, et al. Combined immunodeficiency with EBV positive B cell lymphoma and epidermodysplasia verruciformis due to a novel homozygous mutation in RASGRP1. Clin Immunol (2017) 183:142-4. doi:10.1016/j.clim.2017. 08.007

23. Salzer E, Cagdas D, Hons M, Mace EM, Garncarz W, Petronczki OY, et al. RASGRP1 deficiency causes immunodeficiency with impaired cytoskeletal dynamics. Nat Immunol (2016) 17:1352-60. doi:10.1038/ni.3575

24. Atherly LO, Lucas JA, Felices M, Yin CC, Reiner SL, Berg LJ. The Tec family tyrosine kinases Itk and Rlk regulate the development of conventional CD8+ T cells. Immunity (2006) 25:79-91. doi:10.1016/j.immuni.2006.05.012

25. Blomberg KE, Boucheron N, Lindvall JM, Yu L, Raberger J, Berglof A, et al. Transcriptional signatures of Itk-deficient CD3+, CD4+ and CD8+ T-cells. BMC Genomics (2009) 10:233. doi:10.1186/1471-2164-10-233

26. Broussard C, Fleischacker C, Horai R, Chetana M, Venegas AM, Sharp LL, et al. Altered development of CD8+ T cell lineages in mice deficient for the Tec kinases Itk and Rlk. Immunity (2006) 25:93-104. doi:10.1016/j.immuni. 2006.05.011

27. Dubois S, Waldmann TA, Muller JR. ITK and IL-15 support two distinct subsets of CD8+ T cells. Proc Natl Acad Sci U S A (2006) 103:12075-80. doi:10.1073/pnas.0605212103

28. Nayar R, Enos M, Prince A, Shin H, Hemmers S, Jiang JK, et al. TCR signaling via Tec kinase ITK and interferon regulatory factor 4 (IRF4) regulates CD8+ T-cell differentiation. Proc Natl Acad Sci U S A (2012) 109:E2794-802. doi:10.1073/pnas.1205742109

29. Readinger JA, Mueller KL, Venegas AM, Horai R, Schwartzberg PL. Tec kinases regulate T-lymphocyte development and function: new insights into the roles of Itk and Rlk/Txk. Immunol Rev (2009) 228:93-114. doi:10.1111/ j.1600-065X.2008.00757.x

30. Felices M, Berg LJ. The Tec kinases Itk and Rlk regulate NKT cell maturation, cytokine production, and survival. J Immunol (2008) 180:3007-18. doi:10.4049/jimmunol.180.5.3007

31. Fowell DJ, Shinkai K, Liao XC, Beebe AM, Coffman RL, Littman DR, et al. Impaired NFATc translocation and failure of Th2 development in Itkdeficient CD4+ T cells. Immunity (1999) 11:399-409. doi:10.1016/S10747613(00)80115-6

32. Sun Y, Peng I, Webster JD, Suto E, Lesch J, Wu X, et al. Inhibition of the kinase ITK in a mouse model of asthma reduces cell death and fails to inhibit the inflammatory response. Sci Signal (2015) 8:ra122. doi:10.1126/scisignal.aab0949
33. Graves PE, Siroux V, Guerra S, Klimecki WT, Martinez FD. Association of atopy and eczema with polymorphisms in T-cell immunoglobulin domain and mucin domain-IL-2-inducible T-cell kinase gene cluster in chromosome 5 q 33 . J Allergy Clin Immunol (2005) 116:650-6. doi:10.1016/j.jaci.2005.05.004

34. Kannan AK, Sahu N, Mohanan S, Mohinta S, August A. IL-2-inducible T-cell kinase modulates TH2-mediated allergic airway inflammation by suppressing IFN-gamma in naive CD4+ T cells. J Allergy Clin Immunol (2013) 132:811-20. e1-5. doi:10.1016/j.jaci.2013.04.033

35. Mueller C, August A. Attenuation of immunological symptoms of allergic asthma in mice lacking the tyrosine kinase ITK. J Immunol (2003) 170: 5056-63. doi:10.4049/jimmunol.170.10.5056

36. Miller AT, Berg LJ. Defective Fas ligand expression and activation-induced cell death in the absence of IL-2-inducible T cell kinase. J Immunol (2002) 168:2163-72. doi:10.4049/jimmunol.168.5.2163

37. Gomez-Rodriguez J, Meylan F, Handon R, Hayes ET, Anderson SM, Kirby MR, et al. Itk is required for Th9 differentiation via TCR-mediated induction of IL-2 and IRF4. Nat Commun (2016) 7:10857. doi:10.1038/ncomms10857

38. Gomez-Rodriguez J, Sahu N, Handon R, Davidson TS, Anderson SM, Kirby MR, et al. Differential expression of interleukin-17A and -17F is coupled to T cell receptor signaling via inducible T cell kinase. Immunity (2009) 31:587-97. doi:10.1016/j.immuni.2009.07.009

39. Gomez-Rodriguez J, Wohlfert EA, Handon R, Meylan F, Wu JZ, Anderson SM, et al. Itk-mediated integration of $\mathrm{T}$ cell receptor and cytokine signaling regulates the balance between Th17 and regulatory T cells. J Exp Med (2014) 211:529-43. doi:10.1084/jem.20131459

40. Schaeffer EM, Yap GS, Lewis CM, Czar MJ, McVicar DW, Cheever AW, et al. Mutation of Tec family kinases alters T helper cell differentiation. Nat Immunol (2001) 2:1183-8. doi:10.1038/ni734

41. Schaeffer EM, Debnath J, Yap G, McVicar D, Liao XC, Littman DR, et al. Requirement for Tec kinases Rlk and Itk in T cell receptor signaling and immunity. Science (1999) 284:638-41. doi:10.1126/science.284.5414.638

42. Atherly LO, Brehm MA, Welsh RM, Berg LJ. Tec kinases Itk and Rlk are required for $\mathrm{CD} 8+\mathrm{T}$ cell responses to virus infection independent of their role in CD4+ T cell help. J Immunol (2006) 176:1571-81. doi:10.4049/ jimmunol.176.6.3842

43. Bachmann MF, Littman DR, Liao XC. Antiviral immune responses in Itkdeficient mice. J Virol (1997) 71:7253-7.

44. Kapnick SM, Stinchcombe JC, Griffiths GM, Schwartzberg PL. Inducible $\mathrm{T}$ cell kinase regulates the acquisition of cytolytic capacity and degranulation in CD8+ CTLs. J Immunol (2017) 198:2699-711. doi:10.4049/jimmunol. 1601202

45. Sunil-Chandra NP, Arno J, Fazakerley J, Nash AA. Lymphoproliferative disease in mice infected with murine gammaherpesvirus 68. Am J Pathol (1994) 145:818-26.

46. Chen G, Tai AK, Lin M, Chang F, Terhorst C, Huber BT. Signaling lymphocyte activation molecule-associated protein is a negative regulator of the CD8 T cell response in mice. J Immunol (2005) 175:2212-8. doi:10.4049/ jimmunol.175.4.2212

47. Yin L, Al-Alem U, Liang J, Tong WM, Li C, Badiali M, et al. Mice deficient in the X-linked lymphoproliferative disease gene sap exhibit increased susceptibility to murine gammaherpesvirus-68 and hypo-gammaglobulinemia. J Med Virol (2003) 71:446-55. doi:10.1002/jmv.10504

Conflict of Interest Statement: The authors declare that the research was conducted in the absence of any commercial or financial relationships that could be construed as a potential conflict of interest.

Copyright (C) 2018 Ghosh, Drexler, Bhatia, Adler, Gennery and Borkhardt. This is an open-access article distributed under the terms of the Creative Commons Attribution License (CC BY). The use, distribution or reproduction in other forums is permitted, provided the original author(s) and the copyright owner are credited and that the original publication in this journal is cited, in accordance with accepted academic practice. No use, distribution or reproduction is permitted which does not comply with these terms. 